\title{
Primal-dual active set strategy for large scale optimization of cardiac defibrillation
}

\author{
Nagaiah Chamakuri ${ }^{1 *}$, Karl Kunisch ${ }^{1,2 \dagger}$ \\ ${ }^{1}$ Radon Institute for Computational and Applied Mathematics, \\ Altenbergerstr. 69, Linz, A-4040 Austria. \\ ${ }^{2}$ Institute of Mathematics and Scientific Computing, \\ University of Graz, Heinrichstr. 36, Graz, A-8010 Austria.
}

July 16, 2015

\begin{abstract}
In this paper, the feasible study of the optimal control techniques for the cardiac defibrillation on the anatomical three spatial dimensional rabbit ventricle geometry in the presence of bilateral control constraints. The present work addresses the numerical treatment of multiscale and multidomain simulations of bidomain equations, the description of deriving optimality system, the applicability of primal-dual active set methods to treat the bilateral control constraints for solving such large scale optimization of cardiac defibrillation. The numerical results are demonstrated for the successful defibrillation study on 3D rabbit ventricle geometry by utilizing the less total currents, robustness of the optimization algorithm w.r.t to the variations in the model parameters, feasible study of the multiple smaller boundary control support and the numerical convergence of the optimization algorithm on the finer spatial grids. The parallel efficiency is demonstrated for the primal-dual active set optimization algorithm on such finer spatial grid.
\end{abstract}

Keywords: electrophysiology, cardiac arrhythmia, bidomain model, large scale PDE constraint optimization, control constraints, finite element method, primaldual active set strategy.

\section{Introduction}

In this paper, we present the computational techniques to solve the large scale optimal control problem constrained by set of reaction-diffusion equations along with the presence of control constraints. Specifically, we consider the termination of cardiac arrhythmia in electrophysiology by developing efficient optimal control techniques. Cardiac defibrillation is a standard procedure to restore the regular

\footnotetext{
*nagaiah. chamakuri@ricam. oeaw. ac.at

${ }^{\dagger}$ karl.kunisch@uni-graz.at
} 
heart rhythm in the clinical point of view which delivers the electric shocks through a specified electrodes. The computational tools have been progressively developed to study the cardiac defibrillation and such numerical study has attracted many researchers. Very few journal papers are available for such computational study on anatomical geometry [2, 13, 19, 33, 34].

Here we briefly mention a widely accepted bidomain model [35, 30] which describes the excitation propagation and external stimulation of heart tissue. Here the cardiac tissue domain denoted by $\Omega_{H}$, surrounded bath domain denotes $\Omega_{B}$ and the complete domain denoted by $\Omega=\Omega_{H} \cup \Omega_{B}$. The space-time cylinder of the whole domain is denoted by $Q=\Omega \times[0, T]$ and the cardiac tissue and bath volume are denoted by $Q_{B}=\Omega_{B} \times[0, T]$ and $Q_{H}=\Omega_{H} \times[0, T]$ respectively. Designing of a proper cost functional for the optimization of such complex phenomenon is not trivial. In our computational modeling, a natural optimal control approach to cardiac defibrillation is to determine the control variable in such a way that the undesired values of the transmembrane voltage $v$ are minimized based on the given desired trajectory. The optimal control approach to the cardiac defibrillation is defined as follows:

$$
\left\{\begin{array}{l}
\min J\left(v, I_{e}\right), \\
e\left(u_{b}, u_{e}, v, w, I_{e}\right)=0 \quad \text { in } Q \text { and } I_{e} \in[-R, R],
\end{array}\right.
$$

where $u_{b}, u_{e}, v$ and $w$ are the state variables and $I_{e}$ denotes the control variable of the optimal control problem. Moreover, $R$ is a given maximum threshold value in order to avoid the damaging of the tissue. We considered that the external current $I_{e}$ as control input which acts on the boundary of the bath domain and the transmembrane potential $v$ as one of the state variables which lives on the tissue domain.

The coupled systems of partial differential equations constraints expressed by $e\left(u_{b}, u_{e}, v, w, I_{e}\right)=0$ which consists of the following bidomain equations.

$$
\begin{aligned}
0 & =\nabla \cdot\left(\bar{\sigma}_{i}+\bar{\sigma}_{e}\right) \nabla u_{e}+\nabla \cdot \bar{\sigma}_{i} \nabla v \quad \text { in } Q_{H} \\
\frac{\partial v}{\partial t} & =\nabla \cdot \bar{\sigma}_{i} \nabla v+\nabla \cdot \bar{\sigma}_{i} \nabla u_{e}-I_{i o n}(v, w)+I_{t r}(x, t) \text { in } Q_{H} \\
\frac{\partial w}{\partial t} & =G(v, w) \quad \text { in } Q_{H},
\end{aligned}
$$

where $u_{e}: Q_{H} \rightarrow \mathbb{R}$ is the extracellular potential, $v: Q_{H} \rightarrow \mathbb{R}$ is the transmembrane voltage, $w: Q_{H} \rightarrow \mathbb{R}^{n}$ represents the cell model state variables, $\bar{\sigma}_{i}: \Omega_{H} \rightarrow \mathbb{R}^{d \times d}$ and $\bar{\sigma}_{e}: \Omega_{H} \rightarrow \mathbb{R}^{d \times d}$ are respectively the intracellular and extracellular conductivity tensors. The term $I_{t r}$ is the transmembrane current density stimulus as delivered by the intracellular electrode. Here the transmembrane potential is written as $v=$ $u_{i}-u_{e}, u_{i}$ denotes the intracellular potential. The $I_{i o n}(v, w)$ is the current density flowing through the ionic channels and the function $G(v, w)$ determines the evolution of the gating variables, which are determined by an electrophysiological cell model, see [1] for more description on these models. Here we remark that, the ODE should be solved on each mesh point of computational geometry.

The intra and extra cellular conductivity tensors of the tissue are anisotropic which is a consequence of myocardium geometry. Let $\mathbf{a}_{l}(\mathbf{x}), \mathbf{a}_{t}(\mathbf{x})$ and $\mathbf{a}_{n}(\mathbf{x})$ denotes the fiber, sheet and normal to the sheet directions respectively in the orthonormal basis [19] which depends on the position in the heart. In our computational study, 
we assumed the rotational isotropy at the tissue structure, i.e. $\sigma_{n}^{i, e}=\sigma_{t}^{i, e}$, then the local intracellular conductivity tensor $\bar{\sigma}_{i}$ is expressed as

$$
\bar{\sigma}_{i}=\left(\sigma_{i}^{l}-\sigma_{t}^{i}\right) \mathbf{a}_{l}(\mathbf{x}) \mathbf{a}_{l}^{T}(\mathbf{x})+\sigma_{t}^{i} I,
$$

Here $\sigma_{l}^{i}, \sigma_{t}^{i}$ denote the measured conductivity coefficients along the corresponding directions and $I$ is the identity matrix. The fiber directions of the cardiac tissue have been modeled based on anatomical observations, see Figure 2 for the distributions of the fiber orientation in our computations. A rule-based method was used to impose fiber orientations within the cardiac tissue geometry [5] using fiber angles of $-60^{\circ}$ and $+60^{\circ}$ at the endocardial and epicardial surfaces, respectively, and a smooth linear variation of fiber angles as a function of depth in between.

In general, an additional Poisson problem has to be solved when the heart is immersed in a conductive fluid, e.g. tissue bath in an experimental context or a surrounding torso to model in vivo scenarios.

$$
0=\nabla \cdot \overline{\sigma_{b}} \nabla u_{b} \quad \text { in } Q_{B}
$$

where $u_{b}: Q_{B} \rightarrow \mathbb{R}$ is the bath potential at bath volume and $\overline{\sigma_{b}}: \Omega_{B} \rightarrow \mathbb{R}^{d \times d}$ bath conductivity tensors at bath volume.

In the modeling, assume that there is no current flow between the intracellular and extra-myocardial (bath) domains which accomplish the homogeneous Neumann boundary condition applied to the boundaries on intracellular space.

$$
\eta \cdot\left(\bar{\sigma}_{i} \nabla v+\bar{\sigma}_{i} \nabla u_{e}\right)=0 \quad \text { on } \quad \Sigma_{H}=\partial \Omega_{H} \times[0, T]
$$

The boundary conditions for the extracellular potential on the extracellular domain were set up as a current balance between the extracellular domain and the surrounding bath domain [35. This means, at the boundary of the tissue-bath interface enforce the continuity of the normal component of the extracellular currents $u_{b}$ and $u_{e}$.

$$
\eta \cdot \overline{\sigma_{e}} \nabla u_{e}=\eta \cdot \overline{\sigma_{b}} \nabla u_{b} \text { on } \Sigma_{H}
$$

Here $\eta$ is the outward normal vector on $\partial \Omega_{H}$. Moreover, the extracellular potentials must also match the values at the common boundary.

$$
u_{e}=u_{b} \text { on } \Sigma_{H}
$$

We assume that no current flow out of the bath domain and apply the homogeneous Neumann boundary conditions except for those parts of the boundary where external stimuli are applied, which is injected or withdrawn through electrodes located. We consider that the external stimuli $\hat{I}_{e}(t)$ acts as a control for the optimization problem.

$$
\begin{aligned}
& \eta \cdot \bar{\sigma}_{b} \nabla u_{b}=\hat{I}_{e}(t) \quad \text { on } \quad \Gamma_{12} \times(0, T] \\
& \eta \cdot \bar{\sigma}_{b} \nabla u_{b}=0 \quad \text { on } \partial \Omega_{B} \backslash \Gamma_{12} \times(0, T] .
\end{aligned}
$$

The current $\hat{I}_{e}$ acts as control along the boundary $\Gamma_{12}=\Gamma_{1} \cup \Gamma_{2}$, where $\Gamma_{i}, I=1,2,3$ are mutually disjoint and satisfy $\Gamma_{1} \cup \Gamma_{2} \cup \Gamma_{3}=\partial \Omega_{B}$, see Figure 1 for pictorial representation. For compatibility reasons of Eq. (6) it is assumed throughout that

$$
\int_{\partial \Omega_{B}} \hat{I}_{e}(t, \cdot) d s=0 \quad \forall t \in(0, T) .
$$


In the numerical experiments, $\hat{I}_{e}$ is the only temporally dependent and will take the following form

$$
\hat{I}_{e}=I_{e}(t)\left(\chi_{\Gamma_{1}}-\chi_{\Gamma_{2}}\right)
$$

where $\chi_{\Gamma_{i}}$ is the characteristic function of the set $\Gamma_{i}, i=1,2$. Then condition (13) is satisfied if $\left|\Gamma_{1}\right|=\left|\Gamma_{2}\right|$ where the support regions on $\chi_{\Gamma_{1}}$ and $\chi_{\Gamma_{2}}$ can be considered to represent a cathode and an anode respectively. Due to the pure Neumann elliptic system, we impose zero mean condition for the extracellular potential in order to obtain uniqueness of the elliptic systems.

$$
\int_{\Omega_{H}} u_{e} \mathrm{~d} x=0
$$

In our computations, the initial values of the transmembrane voltage and ion current variables are given by prescribed values.

$$
v(x, 0)=v_{0} \quad \text { and } \quad w(x, 0)=w_{0} \quad \text { on } \Omega_{H},
$$

where $v_{0}: \Omega_{H} \rightarrow \mathbb{R}$ denotes the initial transmembrane potential and $w_{0}: \Omega_{H} \rightarrow \mathbb{R}$ is the initial gating variable at time $t=0$.

The dynamics of the ionic activity at the cell membrane is modeled by a ordinary differential equations which can be solved independently for each node in computations. In our numerical computations, considered the model proposed by Mitchell-Schaeffer(MS) model [26] and modifications by Keener [23]

$$
\begin{aligned}
I_{\text {ion }}(v, w) & =-\frac{1}{\tau_{\text {in }}} w m^{2}(v)\left[v_{p}-v\right]+\frac{1}{\tau_{\text {out }}}\left(v-v_{r}\right) \\
G(v, w) & =\alpha_{w}(v)[1-w]-\beta_{w}(v) w,
\end{aligned}
$$

where

$$
\begin{gathered}
m(v)= \begin{cases}0, & v<v_{r} \\
\frac{v-v_{r}}{v_{p}-v_{r}}, & v_{r}<v<v_{p} \\
1, & \text { else. }\end{cases} \\
\alpha_{w}(v)=\frac{1-f(v)}{\tau_{\text {open }}+\left(\tau_{\text {close }}-\tau_{\text {open }}\right) f(v)} \\
\beta_{w}(v)=\frac{f(v)}{\tau_{\text {open }}+\left(\tau_{\text {close }}-\tau_{\text {open }}\right) f(v)} \\
f(v)=\frac{1}{2}\left(1+\tanh \left(\kappa\left(v-v_{\text {gate }}\right)\right)\right) .
\end{gathered}
$$

Here $v_{r}$ is the resting potential, $v_{p}$ is the peak potential and $v_{\text {gate }}$ change-over voltage. The time constants $\tau_{\text {in }}$ and $\tau_{\text {out }}$ are responsible to the length of the depolarization and repolarization phases where the $\tau_{\text {open }}$ and $\tau_{\text {close }}$ are the characteristic times of gate opening and closing respectively. The above regularized MS model reduces exactly to the original MS model if $\kappa \rightarrow \infty$. Although, the MS ionic model is two variable that keeps the computational complexity as low as possible but on the other hand it produces several action potential features (conduction speed, restitution properties, time scales etc. [26]) at the cardiac cell scale. The parameter values for this model are given in [12]. 
In general, the standard numerical methods are easily incorporated to include anisotropy and inhomogeneity, but the bidomain model equations presents a significant challenge. Large scale computational defibrillation studies involving the bidomain model equations have emerged as a challenge and inevitably leads to a high computational cost due to detailed anatomical geometries of the heart, strongly coupled system of governing model equations, anisotropy of the conductivity tensors, fine scale discretized spatial and temporal domains which are mandatory to capture the reentrant wave propagation and handling the virtual electrode polarization [2, 13, 19, 24, 29, 31, 34, 33]. The numerical treatment of such large multidomain and multiphysics bidomain equations puts significant challenge on the computer memory as well as computational times. Furthermore, requires a appropriate numerical schemes in order to obtain accurate numerical results. In this regard, we have chosen the finite element method for spatial discretization and higher order Rosenbrock time stepping methods for the temporal discretization to solve the PDE system. To perform the large scale simulations of such PDE constrained optimal control problems on realistic geometries is of interest in its own right by utilizing the parallel programming.

It is clear that the state solution is a sub problem associated with the optimization problem. Thus, solving the optimization problem requires more computational time than solving the state equations. Efficient numerical treatment of large scale PDE constrained optimization problem with control constraints poses a significant challenge. The primal-dual active set method has proven to be an efficient numerical method for a large class of PDE constrained optimization problems, see [6, 18, 21, 17, 36]. In [18] shown that the primal-dual active set strategy is equivalent to the semi smooth Newton's method which typically exhibits the local superlinear convergence. The primal-dual active set strategy for nonlinear problems with bilateral control constraints in [20]. We employ such efficient primal-dual active set algorithm for such complex nonlinear model problem under consideration and shown the superlinear convergence in the numerical results.

The objective of the present paper includes 1) The anatomical three spatial dimensional rabbit ventricle geometry is considered for the computational domain. 2) Presents the applicability of the control constraints in order to restrict the external stimulus which could lead to burning or damaging the cardiac tissue. 3) Terminal type cost functional is incorporated which could speed up the optimization algorithm. 4) The primal-dual active set method is adopted to solve the optimization algorithm in order to handle the bilateral control constraints, 5) Investigated the robustness of the optimization algorithm w.r.t the model parameters on the realistic 3D heart geometry and studied the smaller locally supported control domains at the boundary of the bath domain.

The remaining of the paper is organized as follows: In the next subsection we provide the mathematical review of the bidomain-bath model equations which are needed in the optimal control framework. In Section 2, we provide the optimal control framework for the cardiac defibrillation and the primal-dual active set algorithm to solve the optimality system in the presence of control constraints is described in subsection 2.1. The brief numerical procedure to solve the primal and dual equations and the optimization algorithmic procedure is given in Section 3 . In Section 4. the computational setup and numerical results for the successful defibrillation is demonstrated. The different numerical test cases are discussed for robustness of the optimization algorithm. Finally, we discuss the summary of the presented work. 


\subsection{Analytical background}

We denote $\Omega_{H}, \Omega_{B} \subset \mathbb{R}^{3}$ are bounded domains with Lipschitz boundaries $\partial \Omega_{H}$ and $\partial \Omega_{B}$ respectively. The fixed time horizon denoted by $T>0$. We denote

$$
\begin{gathered}
H=L^{2}(\Omega), \quad V=H^{1}\left(\Omega_{H}\right) \\
U=\left\{u \in H^{1}(\Omega): \int_{\Omega_{H}} u \mathrm{~d} x=0\right\}, \quad U_{e}=\left\{u \in H^{1}\left(\Omega_{H}\right): \int_{\Omega_{H}} u \mathrm{~d} x=0\right\} .
\end{gathered}
$$

Here assume that the intra cellular, extra cellular and the bath conductivity tensors $\sigma_{i}, \sigma_{e}$ are symmetric, positive definite matrix with the coefficients in $L^{\infty}$ i.e. there exist $0<m<M$

$$
m|\xi|^{2} \leq \xi^{T} \sigma_{i, e, b} \xi \leq M|\xi|^{2}, \quad \text { for all } \xi \in \mathbb{R}^{3} .
$$

For the analysis, we assume that the resting potential is zero and the peak potential is 1 . The reaction terms of the membrane model can be written as follows.

$$
\begin{aligned}
I_{\text {ion }}(v, w) & =f_{1}(v) w+f_{2}(v) \\
G(v, w) & =g_{1}(v) w+g_{2}(v) .
\end{aligned}
$$

where $f_{1}(v)=-\frac{1}{\tau_{i n}} w v^{2}(1-v)$ and $f_{2}(v)=\frac{v}{\tau_{\text {out }}}$. For brevity, we denote the following spaces corresponding to the state solution.

$$
\begin{gathered}
X_{1}=L^{2}(0, T ; U) \quad X_{2}=C^{0}(0, T ; H) \cap L^{2}(0, T ; V) \cap L^{4}\left(Q_{H}\right) \\
X_{3}=W^{1, \infty}\left(0, T ; L^{\infty}(Q)\right) \quad X_{4}=L^{\infty}(0, T ; \mathbb{R}) .
\end{gathered}
$$

The weak solution of the Eqs. (2)-(4) on $\Omega_{H}$ is given by

$$
\begin{aligned}
\int_{\Omega_{H}} \bar{\sigma}_{i} \nabla v(t) \nabla \varphi \mathrm{d} x+\int_{\Omega_{H}}\left(\bar{\sigma}_{i}+\bar{\sigma}_{e}\right) \nabla u_{e}(t) \nabla \varphi \mathrm{d} x & =0 \\
\left\langle v_{t}(t), \psi\right\rangle_{V^{*}, V}+\int_{\Omega_{H}} \bar{\sigma}_{i} \nabla\left(u_{e}(t)+v(t)\right) \nabla \psi d x & +\int_{\Omega_{H}} I_{i o n}(v(t), w(t)) \psi d x \\
& =\left\langle I_{t r}(t), \psi\right\rangle_{V^{*}, V} \\
\left\langle w_{t}(t), \chi\right\rangle_{V_{H}^{*}, V_{H}}-\int_{\Omega_{H}} G(v(t), w(t)) \chi d x & =0
\end{aligned}
$$

together with initial and boundary conditions (7)-(16) and for all $(\varphi, \psi, \chi) \in\left(U_{e}, V, H\right)$. Let $V^{h} \subset H^{1}\left(\Omega_{H}\right)$ be the finite dimensional subspace of piecewise linear basis functions with respect to the spatial grid. We considered that the elliptic system on the tissue domain Eq. (2) and on bath domain Eq. (2) can be solved monolithically. For this purpose we define $u$ as the extracellular potential on $\Omega_{H} \cup \Omega_{B}$, i.e.:

$$
u= \begin{cases}u_{b} & \text { in } \Omega_{B} \\ u_{e} & \text { in } \Omega_{H}\end{cases}
$$

and the similar way we can define the global conductivity tensor as follows.

$$
\bar{\sigma}= \begin{cases}\bar{\sigma}_{b} & \text { in } \Omega_{B} \\ \left(\bar{\sigma}_{i}+\bar{\sigma}_{e}\right) & \text { in } \Omega_{H}\end{cases}
$$


Using the above definitions, the weak solution of the elliptic system on the integrated domain $\Omega$ reads as follows.

$$
\int_{\Omega_{H}} \bar{\sigma}_{i} \nabla v(t) \nabla \varphi_{b} \mathrm{~d} x+\int_{\Omega} \bar{\sigma} \nabla u(t) \nabla \varphi_{b} \mathrm{~d} x=\int_{\Gamma_{12}} I_{e}(t, \cdot) \varphi_{b} \mathrm{~d} s
$$

Definition 1.1. If $I_{e} \in L^{2}\left(\partial \Omega_{B} \times(0, T)\right), I_{t r} \in L^{2}\left(\Omega_{H} \times(0, T)\right)$,

$$
\overline{\sigma_{i}} \in L^{\infty}\left(\Omega_{H}\right)^{d \times d}, \overline{\sigma_{e}} \in L^{\infty}\left(\Omega_{H}\right)^{d \times d}, \overline{\sigma_{b}} \in L^{\infty}\left(\Omega_{B}\right)^{d \times d},
$$

are symmetric and uniformly elliptic, and if $v_{0} \in V_{H}$, and $w_{0} \in L^{\infty}\left(\Omega_{H}\right)$, with $r<w_{0} \leq 1$ in $\Omega_{H}$, for some $r>0$, then a triple $(u, v, w) \in X_{1} \times X_{2} \times X_{3}$ is called the weak solution of the bidomain system (2)-(4), (6) iff the functions $u, v, w$ satisfy the variation formulation (27)-(29).

Proposition 1.2 (A-priori estimates). If a triple $(u, v, w) \in\left(X_{1} \times X_{2} \times X_{3}\right)$ satisfy a weak solution of the bidomain equations (27)-(29) and satisfy the initial solution of the state system $v_{0} \in L^{2}\left(\Omega_{H}\right), w_{0} \in L^{\infty}\left(\Omega_{H}\right)$ then the following a-priori error estimates holds:

$$
\begin{gathered}
\|u\|_{L^{2}(0, T ; U)}+\|v\|_{C^{0}(0, T ; H)}+\|v\|_{L^{2}\left(0, T ; H^{1}\left(\Omega_{H}\right)\right)}+\|v\|_{L^{4}(Q)}+\left\|v_{t}\right\|_{L^{p^{\prime}}\left(0, T ; V^{*}\right)} \\
+\|w\|_{X_{3}}+\left\|w_{t}\right\|_{L^{2}(0, T ; H)} \leq C\left(\left\|v_{0}\right\|+\left\|w_{0}\right\|+\left\|I_{i t r}\right\|_{L^{2}\left(V^{*}\right)}+\left\|I_{e}\right\|_{X_{4}}\right)
\end{gathered}
$$

For the proof we referred to [9]. Here the estimates were derived for the tissuetorso model by using the parabolic-parabolic form of the bidomain model.

\section{Optimal control formulation}

In this section we are concerned with the optimal control of cardiac defibrillation where the external current acts as a control which presents at the boundary of the surrounded bath domain. We propose the formulation of the optimal control formulation for the successful cardiac defibrillation. We derive the first and second order optimality conditions.

Specifically, in our computations, the cardiac defibrillation aims at driving the transmembrane voltage $v$ to a desired value denoted by $v_{d}$ at the intracellular space by properly applying the $I_{e}$ at the boundary of bath domain. The optimal control problem suitable to optimize the irregular heart rhythm is formulated as follows.

$$
\left\{\begin{array}{l}
\min J\left(v, I_{e}\right)=J_{1}(v)+J_{2}\left(I_{e}\right) \\
\text { subject to } 2-6) \text { and } I_{e} \in \mathcal{U}_{\mathrm{ad}}
\end{array}\right.
$$

where

$$
\begin{aligned}
J_{1}(v) & =\frac{\alpha_{1}}{2} \int_{0}^{T} \int_{\Omega_{H}}\left|v-v_{d}\right|^{2} \mathrm{~d} x \mathrm{~d} t+\frac{\alpha_{3}}{2} \int_{\Omega_{H}}\left|v(T)-v_{d}(T)\right|^{2} \mathrm{~d} x \\
J_{2}\left(I_{e}\right) & =\frac{\alpha_{2}}{2} \int_{0}^{T}\left|I_{e}(t)\right|^{2} \mathrm{~d} t
\end{aligned}
$$

Here $\alpha_{1}$ and $\alpha_{3}$ are the weights at the observation domain, $\alpha_{2}$ is the weight of the cost of the control. In order to avoid high energy at the tissue we impose the 
bounds on the control data. The set of admissible controls $U_{a d}$ is prescribed by the following bilateral pointwise constraints.

$$
\mathcal{U}_{\mathrm{ad}}=\left\{I_{e} \in L^{\infty}(0, T ; \mathbb{R}) \mid-R \leq I_{e}(t) \leq R \text { for a.e. } t \in(0, T)\right\},
$$

for any given constant $R \in \mathbb{R}$. We assume that the control domain $\mathcal{U}_{\text {ad }} \in L^{\infty}(0, T ; \mathbb{R})$ is bounded, convex and closed. We point out that, for any feasible control $I_{e} \in \mathcal{U}_{\text {ad }}$ there exist a weak solution $\left(u_{b}, u_{e}, v, w\right)$ of the bidomain model by the proposition 1.2 . Consequently, the control-to-state mapping is well defined $\mathcal{U}_{\text {ad }} \ni I_{e} \longmapsto$ $(u, v, w) \in X_{1} \times X_{2} \times X_{3}$. We assume that $J_{1}(v): X_{2} \rightarrow \mathbb{R}$ is weakly lower semi-continuous.

Proposition 2.1 (Existence of a global optimal solution). There exists at least one global minimizer for the optimal control problem 31.

Proof. Let us define the following

$$
S_{a d}=\left\{\left(u, v, w, I_{e}\right) \in X_{1} \times X_{2} \times X_{3} \times X_{4} ; e\left(u, v, w, I_{e}\right)=0\right\} .
$$

From the existence results for the bidomain-bath equations, we can obtain that there exists a $\left(u, v, w, I_{e}\right) \in X_{1} \times X_{2} \times X_{3} \times X_{4}$. Consequently, $\left(u, v, w, I_{e}\right) \in S_{a d}$ and $S_{a d} \neq 0$.

From the additional assumption on the cost functional $J$ that the $J$ is bounded from below $\frac{\alpha_{2}}{2}\left\|I_{e}\right\|^{2} \leq J\left(v, I_{e}\right)<\infty$. Then there exists a minimizing sequence $\left\{I_{e}^{n}\right\}$ that is uniformly bounded in $X_{4}$. Let denote the associated state variables by $u^{n}, v^{n}, w^{n}$. By Proposition 1.2 , there exist a subsequence $\left(u^{*}, v^{*}, w^{*}, I_{e}^{*}\right) \in X_{1} \times$ $X_{2} \times X_{3} \times X_{4}$ and $v_{t} \in L^{2}\left(Q_{H}\right), w_{t} \in L^{2}\left(Q_{H}\right)$. Since $\left\{I_{e}^{n}\right\}$ is bounded in $X_{4}$, the subsequence $\left\{u^{n}, v^{n}, w^{n}\right\}$ is also uniformly bounded. Consequently, we can extract the following convergent sequence

$$
\begin{gathered}
I_{e}^{n} \rightarrow I_{e}^{*} \text { in } X_{4} \text { as } n \rightarrow \infty, \\
u^{n} \rightarrow u^{*} \text { in } X_{1} \text { as } n \rightarrow \infty, \\
v^{n} \rightarrow v^{*} \text { in } X_{2} \text { as } n \rightarrow \infty, \\
w^{n} \rightarrow w^{*} \text { in } X_{3} \text { as } n \rightarrow \infty .
\end{gathered}
$$

The set $\mathcal{U}_{\text {ad }}$ is convex and closed in $X_{4}$ and consequently $I_{e}^{*}$ satisfies also the control constraints. It remains to argue that $\left(u^{*}, v^{*}, w^{*}, I_{e}^{*}\right)$ is a solution to the bidomain-bath equations by passing to the limit in the equations $\left(u^{n}, v^{n}, w^{n}, I_{e}^{n}\right)$. This can be realized as in [9, Section 3.4]. Additional assumption on the $J_{i}$ imply that $J$ is weakly lower semi-continuous and we have the following result.

$$
J\left(v^{*}, I_{e}^{*}\right) \leq J\left(v^{n}, I_{e}^{n}\right) .
$$

Now we turn to derive the optimality system. In this regard, the Lagrangian related to the optimal control problem is defined by

$$
\mathcal{L}\left(u_{b}, u_{e}, v, w, I_{e}, p_{b}, p, q, r\right)=J\left(v, I_{e}\right)+\left\{e\left(u_{b}, u, v, w, I_{e}\right),\left(p_{b}, p, q, r\right)\right\}
$$


where $p_{b}, p_{e}, q, r$ are the Lagrange multipliers associated to the state Eqs. (6)-(4). More, specifically the Lagrangian corresponding to Eq. (31) to obtain the first and second order optimality conditions is given as follows.

$$
\begin{aligned}
& \mathcal{L}\left(u_{b}, u_{e}, v, w, I_{e}, p_{b}, p, q, r\right)=J\left(v, I_{e}\right) \\
& -\int_{0}^{T} \int_{\Omega} \sigma_{b} \nabla u_{b} \nabla p_{b}+\int_{0}^{T} \int_{\Gamma_{12}} I_{e} p_{b} \mathrm{~d} s \mathrm{~d} t \\
& -\int_{0}^{T} \int_{\Omega_{H}}\left(\sigma_{i} \nabla v \nabla p_{e}+\left(\sigma_{i}+\sigma_{e}\right) \nabla u_{e} \nabla p_{e}\right) \mathrm{d} x \mathrm{~d} t \\
& -\int_{0}^{T} \int_{\Omega_{H}}\left(v_{t} q+\sigma_{i}(\nabla u+\nabla v) \nabla q+I_{i o n}(v, w) q-I_{t r} q\right) \mathrm{d} x \mathrm{~d} t \\
& -\int_{0}^{T} \int_{\Omega_{H}} w_{t} r-G(v, w) r \mathrm{~d} x \mathrm{~d} t,
\end{aligned}
$$

The first order optimality system is given by the Karusch-Kuhn-Tucker (KKT) conditions. For this purpose the partial derivatives of $\mathcal{L}$ with respect to $u_{b}, u_{e}, v$ and $w$ are set equal to zero. Then we obtain the following dual equations.

$$
\begin{aligned}
0 & =\nabla \cdot\left(\bar{\sigma}_{i}+\bar{\sigma}_{e}\right) \nabla p_{e}+\nabla \cdot \bar{\sigma}_{i} \nabla q \text { in } Q_{H}, \\
\frac{\partial q}{\partial t} & =-\nabla \cdot \bar{\sigma}_{i} \nabla p_{e}-\nabla \cdot \bar{\sigma}_{i} \nabla q+\left(I_{i o n}\right)_{v} q-(G)_{v} r-\left.\left(V_{m}-V_{d}\right)\right|_{\Omega_{o b s}} \text { in } Q_{H}, \\
\frac{\partial r}{\partial t} & =\left(I_{i o n}\right)_{w} q-(G)_{w} r \text { in } Q_{H}, \\
0 & =\nabla \cdot \bar{\sigma}_{b} \nabla p_{b} \quad \text { in } Q_{B}
\end{aligned}
$$

where $p_{b}, p_{e}, q$ and $r$ are the Lagrange multipliers associated to $u_{b}, u_{e}, v$ and $w$ respectively. The terminal conditions are

$$
q(T)=\alpha_{3}\left(v(T)-v_{d}(T)\right), \quad r(T)=0,
$$

and the boundary conditions for the dual states must satisfy

$$
\begin{aligned}
\eta \cdot\left(\bar{\sigma}_{i} \nabla p_{e}+\bar{\sigma}_{i} \nabla q\right)=0 & \text { on } \Sigma_{H} \\
\overline{\sigma_{b}} \nabla p_{b} \cdot \eta=0 & \text { on } \partial \Omega_{B} \times[0, T] .
\end{aligned}
$$

Furthermore, the following interface conditions should satisfy.

$$
p_{b}=p_{e} \text { and } \sigma_{i} \nabla p_{e}=\sigma_{b} \nabla p_{b} \text { on } \Sigma_{H} \text {. }
$$

In addition the zero mean conditions $\int_{\Omega_{B}} p_{b}(t) d x=0$ and $\int_{\Omega_{H}} p_{e}(t) d x=0$ holds for all $t \in(0, T)$. Finally we have the optimality condition:

$$
\left(\alpha_{2} I_{e}^{*}(t)+\int_{\Gamma_{1}} p_{b}(x, t) d x-\int_{\Gamma_{2}} p_{b}(x, t) d x\right)\left(I_{e}(t)-I_{e}^{*}(t)\right) \geq 0 \quad \text { for all } I_{e} \in U
$$

for almost every $t \in(0, T)$, which, in case the constraints are not active becomes

$$
\alpha_{2} I_{e}^{*}(t)+\int_{\Gamma_{1}} p_{b}(x, t) d x-\int_{\Gamma_{2}} p_{b}(x, t) d x=0 \text {, for a.e. } t \in(0, T) .
$$




\subsection{Primal-Dual active set method}

The well known primal-dual active set strategy [6, 17, 20] is explained for the optimal control of bidomain equations together with the bilateral control constraints in this section.

We define the solution operator $S: L^{\infty}(0, T ; \mathbb{R}) \rightarrow X_{1} \times X_{2} \times X_{3} \times X_{4}$ by $\left(u_{b}, u, v, w\right)=S\left(I_{e}\right)$ for $I_{e} \in L^{\infty}(0, T ; \mathbb{R})$ and $\left(u_{b}, u_{e}, v, w\right)$ is the solution to Eqs. (2)(6). Introducing the reduced cost functional

$$
\hat{J}\left(I_{e}\right)=J\left(S\left(I_{e}\right), I_{e}\right)
$$

and the problem $(\mathrm{P})$ can be expressed as

$$
\left\{\begin{array}{l}
\min \hat{J}\left(I_{e}\right), \\
\text { s.t. } u \in \mathcal{U}_{\mathrm{ad}}
\end{array}\right.
$$

Observe that $(\hat{\mathrm{P}})$ is a minimization problem with bilateral constraints but with no equality constraints. The gradient of $\hat{J}\left(I_{e}\right)$ at fixed point $I_{e} \in L^{\infty}(0, T ; \mathbb{R})$ is defined as

$$
\nabla \hat{J}\left(I_{e}\right)=\alpha_{2} I_{e}(t)+\int_{\Gamma_{1}} p_{b}(x, t) d x-\int_{\Gamma_{2}} p_{b}(x, t) d x
$$

where $\left(p_{b}, p_{e}, q, r\right)$ solve the dual equations for the state solution pair $\left(u_{b}, u_{e}, v, w\right)$ which is a solution to Eqs. (2)-(6) for the given control $I_{e} \in L^{\infty}(0, T ; \mathbb{R})$.

Here we recall the definition of Newton-differentiability which is useful in the following. Let $X, Y$ are Banach spaces and $D \subset X$ is an open subdomain in $X$. Let $F: D \rightarrow Y$ be a nonlinear mapping and $\mathcal{L}(X, Y)$ be the set of continuous linear mappings from $X$ to $Z$. Assume that the nonlinear operator equation as follows,

$$
F(x)=0
$$

Definition 2.2. The mapping $F: D \rightarrow Y$ is called Newton differentiable on the open subset $U \subset D$ if there exists a family of generalized derivatives $G: U \rightarrow$ $\mathcal{L}(X, Y)$ such that

$$
\lim _{h \rightarrow 0} \frac{1}{\|h\|}\|F(x+h)-F(x)-G(x+h) h\|_{Y}=0 \quad \forall \quad x \in U .
$$

Consider the Newton differentiability of the pointwise max-operator. Lets denote the $\max$ operator by $\max (0, u)$ for $u \in X$. For $\delta \in \mathbb{R}$ we introduce the following functions for its generalized derivative of the form,

$$
G(u)(x)= \begin{cases}1 & \text { if } u(x)>0 \\ \delta & \text { if } u(x)=0 \\ 0 & \text { if } u(x)<0\end{cases}
$$

The proof of the following proposition can find in [18].

Proposition 2.3. The mapping $\max (0,):. L^{q}(\Omega) \rightarrow L^{r}(\Omega)$ with $1 \leq r<q \leq \infty$ is Newton differentiable on $L^{q}$ and $G_{\delta}$ is a generalized derivative.

We refer to [18] for the proof. 
Proposition 2.4. The first order necessary optimality conditions for the (44) can be expressed as the variational inequality

$$
\left\langle\nabla \hat{J}\left(I_{e}\right),\left(\bar{I}_{e}(t)-I_{e}(t)\right)\right\rangle \geq 0 \quad \text { for all } \bar{I}_{e} \in U
$$

This can be equivalently expressed as an optimality system for the control $I_{e} \in \mathcal{U}_{\text {ad }}$ and the corresponding multiplier $\eta \in \mathcal{U}_{a d}$ as

$$
\begin{aligned}
& \bar{I}_{e}(t)+\eta=0 \\
& \eta=\max \left(0, \eta+c\left(I_{e}-R\right)\right)+\min \left(0, \eta+c\left(I_{e}+R\right)\right)
\end{aligned}
$$

where max and min are interpreted as pointwise sense.

The proof is straightforward based on the convexity properties of the admissible control set $\mathcal{U}_{\text {ad }}$.

Note that the operator equation (48) is Lipschitz continuous but not differentiable in the classical sense. In spite of that, it enjoys a property called Newton differentiability that generalizes the concept of differentiability in the case of $c=\alpha_{2}$, see for more details in [21, 18]. Other choices $c \neq \alpha_{2}$ were discussed in [6, 21]. Now we define the following active and inactive sets at the $k$ th Newton iteration.

$$
\begin{aligned}
& \mathcal{A}_{+}^{k}=\left\{t \in[0, T]: I_{e}^{k}(t)+\frac{\eta^{k}}{c} \leq-R\right\}, \\
& \mathcal{A}_{-}^{k}=\left\{t \in[0, T]: I_{e}^{k}(t)+\frac{\eta^{k}}{c} \geq R\right\}, \\
& \mathcal{A}=\mathcal{A}_{+} \cup \mathcal{A}_{-}, \quad \text { and } \quad \mathcal{I}^{k}=[0, T] \backslash \mathcal{A} .
\end{aligned}
$$

With the above settings, the Newton iteration step is formulated as follows

$$
\left(\begin{array}{cc}
\hat{J}^{\prime \prime}\left(I_{e_{k}}\right) & \chi_{\mathcal{A}} \\
\chi_{\mathcal{A}} & 0
\end{array}\right)\left(\begin{array}{c}
\delta I_{e} \\
\delta \eta_{\mathcal{A}^{k}}
\end{array}\right)=\left(\begin{array}{c}
\hat{J}^{\prime}\left(I_{e_{k}}\right)+\chi_{\mathcal{A}} \eta_{k} \\
\chi_{\mathcal{A}_{+}}\left(I_{e_{k}}-R\right)+\chi_{\mathcal{A}_{-}}\left(I_{e_{k}}+R\right)
\end{array}\right)
$$

where $\chi_{X}$ denotes the characteristic function of a set $X$ and $\delta \eta_{\mathcal{A}^{k}}$ denotes the restriction of the active set $\mathcal{A}_{k}$

The complete details of primal-dual active set algorithm is given in Algorithm 2 ,

\section{$3 \quad$ Numerical approach}

In this section, we introduce the spatial and temporal discretization of the primal and dual equations.

\section{Space discretization of the primal problem}

We give an overview of the spatial discretization of the bidomain equations by a piecewise bilinear finite element method in this subsection. As we mentioned that the elliptic system is solved as a monolithic approach. For the spatial discretization of the bidomain equations, we consider the weak formulation (27)-(29). In this regard, the approximate solutions of the vectors $\mathbf{u}, \mathbf{v}$ and $\mathbf{w}$ are expressed in the form $\mathbf{u}(t)=$ $\sum_{i=0}^{M+N} u_{i}(t) \omega_{i}, \mathbf{v}(t)=\sum_{i=0}^{N} v_{i}(t) \omega_{i}$ and $\mathbf{w}(t)=\sum_{i=0}^{N} w_{i}(t) \omega_{i}$, respectively, where 
$\left\{\omega_{i}\right\}_{i=1}^{M}$ and $\left\{\omega_{i}\right\}_{i=1}^{N}$ denote the basis functions. This semi-discretization in space results in the following matrix representation of the differential algebraic system.

$$
\begin{aligned}
\mathbf{A}_{\mathbf{i e}} \mathbf{u}+R_{H} \mathbf{A}_{\mathbf{i}} \mathbf{v} & =\mathbf{I}_{e} \\
\mathbf{M} \frac{\partial \mathbf{v}}{\partial t} & =-\mathbf{A}_{\mathbf{i}} \mathbf{v}-R_{B} \mathbf{A}_{\mathbf{i}} \mathbf{u}-\mathbf{I}_{i o n}(\mathbf{v}, \mathbf{w})+\mathbf{I}_{t r} \\
\mathbf{M} \frac{\partial \mathbf{w}}{\partial t} & =\mathbf{G}(\mathbf{v}, \mathbf{w})
\end{aligned}
$$

together with initial conditions for $\mathbf{v}$ and $\mathbf{w}$, where $\mathbf{A}_{\mathbf{i e}}=\left\{\left\langle\left(\sigma_{i}+\sigma_{e}\right) \nabla \omega_{i}, \nabla \omega_{j}\right\rangle\right\}_{i, j=1}^{M+N}$ and $\mathbf{A}_{\mathbf{i}}=\left\{\left\langle\sigma_{i} \nabla \omega_{i}, \nabla \omega_{j}\right\rangle\right\}_{i, j=1}^{N}$ are the stiffness matrices, $\mathbf{M}=\left\{\left\langle\omega_{i}, \omega_{j}\right\rangle\right\}_{i, j=1}^{N}$ is the mass matrix, the vectors $\mathbf{I}_{e}, \mathbf{I}_{i t r}$ are defined by $\mathbf{I}_{e}=\left\{\left\langle\left(\chi_{\Gamma_{1}} I_{e}-\chi_{\Gamma_{2}} I_{e}\right), \omega_{j}\right\rangle\right\}_{j=1}^{M_{\Gamma}}$ and $\mathbf{I}_{t r}=\left\{\left\langle I_{t r}, \omega_{j}\right\rangle\right\}_{j=1}^{N}$, respectively. Here the $R_{H}$ and $R_{B}$ represent the restriction operator from the tissue domain to the integrated domain and from the integrated domain to the tissue domain respectively. The expressions $\left(\mathbf{I}_{i o n}\right)(\mathbf{v}, \mathbf{w})$ and $\mathbf{G}(\mathbf{v}, \mathbf{w})$ are defined by

$$
\begin{aligned}
\left(\mathbf{I}_{i o n}\right)(\mathbf{v}, \mathbf{w}) & =\left\{\left\langle I_{i o n}\left(\sum_{i=0}^{N} v_{i} \omega_{i}, \sum_{i=0}^{N} w_{i} \omega_{i}\right), \omega_{j}\right\rangle\right\}_{j=1}^{N}, \\
\mathbf{G}(\mathbf{v}, \mathbf{w}) & =\left\{\left\langle G\left(\sum_{i=0}^{N} v_{i} \omega_{i}, \sum_{i=0}^{N} w_{i} \omega_{i}\right), \omega_{j}\right\rangle\right\}_{j=1}^{N} .
\end{aligned}
$$

Remark 3.1. Analogously, we follow the piecewise bilinear finite element method for the spacial discretization of the dual equations and we obtain similar matrix representation system as in Eqs. (51)-(53). Moreover, we employ the same spatial discretization technique for the linearized primal and dual equations.

Remark 3.2. We mention that the compatibility condition for the singular algebraic system Eq. (51) is satisfied at each time iteration level, i.e.

$$
\mathbf{c}^{T}\left(-R_{H} \mathbf{A}_{\mathbf{i}} \mathbf{v}-\mathbf{I}_{e}\right)=-\left(\mathbf{c}^{T} R_{H} \mathbf{A}_{\mathbf{i}}\right) \mathbf{v}-\mathbf{c}^{T} \mathbf{I}_{e}=0
$$

where $\mathbf{c}=(1, \ldots, 1)^{T}$ is a constant vector and we take $\mathbf{u}_{0}$ has zero mean. Similarly, at every time-level the iterative procedure for solving the elliptic system in dual, linearized primal and linearized dual systems is initialized by a zero-mean function in order to satisfy the compatibility condition.

\section{Time discretization}

In this subsection we give a brief description of the time discretization for solving the systems of ordinary differential equations. The time discretization of the bidomain equations can be performed using either implicit, semi-implicit or explicit schemes and for a detailed comprehensive study on the stability and accuracy of several bidomain time discretizations we refer to the work in [15]. In our computational approach, the semi discretized Eqs. (52) and (53) solved as a coupled system which can be expressed in the following matrix representation.

$$
\tilde{\mathbf{M}} \frac{\partial \mathbf{x}}{\partial t}=\mathbf{F}(\mathbf{x}), \quad \mathbf{x}\left(t^{0}\right)=\mathbf{x}^{\mathbf{0}} .
$$


where

$$
\begin{aligned}
\tilde{\mathbf{M}} & =\left(\begin{array}{cc}
\mathbf{M} & 0 \\
0 & \mathbf{M}
\end{array}\right), \quad \mathbf{x}=\left(\begin{array}{c}
\mathbf{v} \\
\mathbf{w}
\end{array}\right), \\
\mathbf{F} & =\left(\begin{array}{c}
-\mathbf{A}_{\mathbf{i}} \mathbf{v}-R_{B} \mathbf{A}_{\mathbf{i}} \mathbf{u}-\mathbf{I}_{i o n}(\mathbf{v}, \mathbf{w})+\mathbf{I}_{t r} \\
\mathbf{G}(\mathbf{v}, \mathbf{w})
\end{array}\right)
\end{aligned}
$$

To solve (54), we introduce discrete steps $0=t^{0}, t^{1}, \ldots, t^{n}=T$ in the time interval $[0, T]$ which are not necessarily equidistant. We further set $\tau^{i}=t^{i+1}-t^{i}$ and denote by $\mathbf{x}^{i}$ the numerical solution at time $t^{i}$. In our computations, we used the linearly implicit Runge-Kutta methods for the time discretization and they serves as a good compromise between stability and efficiency, see in [16, 10] which require at each time step the solution of 24 linear systems. These belong to a large class of methods which try to avoid the nonlinear system and replace it by a sequence of linear ones. In simulations, we used exact derivatives of the vector $\mathbf{F}(\mathbf{x})$ for the construction of the Jacobian matrix. For our computations the ROWDA method, see [25], was employed which has three internal stages to solve in each time step. Here we avoid the time discretization details for the current problem which was well explained in [10, Section 3.2].

\section{Computer implementation}

In a nutshell, we explain the solution procedure to solve the bidomain equations and optimization problem. In our computations, the public domain FEM software package DUNE [3], especially the dune-pdelab [4] discretization module, was used which is a $\mathrm{C}++$ template based programming environment. For construction of the parallel grids, the ALUGrid library [14] was employed which in turn uses the METIS 22] graph partitioner for the decomposition of the grid.

First we give a brief description of numerical techniques to solve the primal system and analogously, we can employ similar techniques to solve the dual, linearized primal and linearized dual equations. The solution of the singular linear systems of Eq. (51) is defined up to an additive constant and to fix this constant we impose a zero mean condition. For the numerical realization of this condition together with the PDEs one needs a special purpose numerical methods to solve the system uniquely. For this purpose, we adopted a stabilized saddle point formulation from the work of Bochev and Lehoucq [8], see [10, 11] for the discussion and implementation details of this technique for the current problem. After the full discretization of the PDEs we obtain a system of linear algebraic equations and to solve the linear system we employed a Conjugate Gradient (CG) method with AMG preconditioner [7, which is developed using a greedy heuristic algorithm for the aggregation based on a strength of connection criterion. We used a BiCGSTAB method with Jacobi preconditioning to solve the linear algebraic system which arrived after the time discretization of (54) at each internal stage of the ROWDA method.

Due to the presence of two different grids, the numerical treatment of the bidomain equations is not a trivial task. As mentioned, we solve the combined elliptic system Eq. 51 on the integrated domain $\Omega$ while the parabolic-ode system Eq. 54 needs to be solved on the cardiac tissue domain $\Omega_{H}$ only. Realization of our simulations based on the loading two grid data structures, one for the whole domain and a second one for embedded tissue domain, separately. In parallel computations, two distinct non-overlapping grids constructed, decomposition based on the METIS 
graph partitioner, to solve the PDE system. Every single processor is assigned to two different subdomains and inter-communication between the processors is necessary at each time step. To accomplish the necessary communication a projection operator, realized as a generalized scattering operation, was used to map between the parallel data vectors $V_{m}$ and $\phi_{e}$. In Algorithm 1 , we briefly explain the numerical strategy to solve the primal system which is a fundamental step in the optimization procedure.

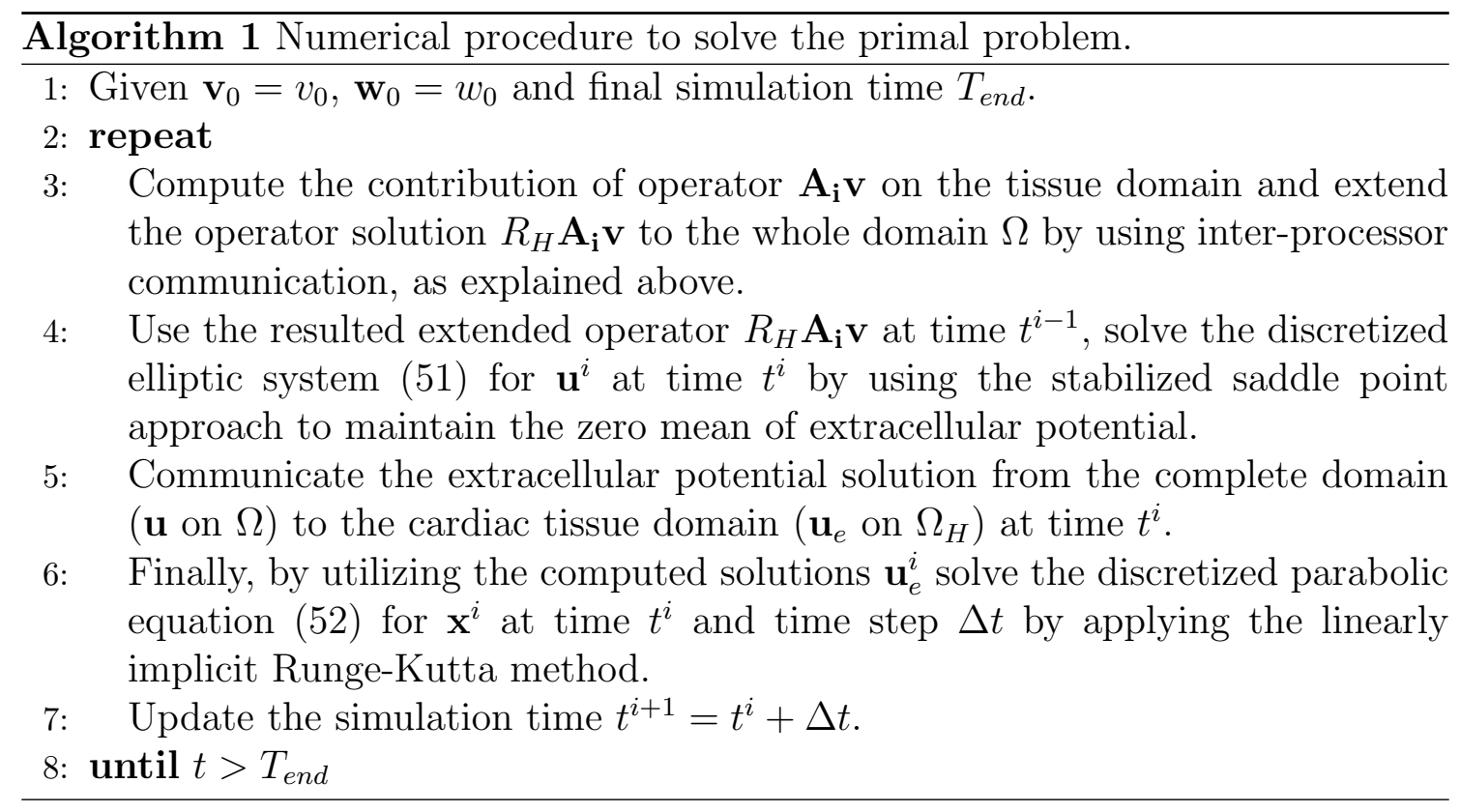

As explained, we employ the primal-dual active set method for solving the optimization problem with the presence of control constraints, the Algorithm steps are given in 2. Briefly, here we discuss the computational complexity of the primal dual active set method. First, we recall the number of PDE solves that are need for optimization. The Newton method requires the evaluation of the Hessian of the reduced cost functional, step 8 in Algorithm 2, in direction of the increment of the control $\delta I_{e}$ which is required for any iterative Krylov solver. Each inner iteration is achieved by one linearized primal and a dual equation solver in order to achieve the matrix-vector product of the Hessian on $\delta I_{e}$. The solution of these linearized equations in parallel was done similarly to the solution procedure for primal equations. Here is a brief outline of complete a Newton's optimization algorithm with primal-dual active set strategy.

\section{Numerical results}

In this section, the numerical results are demonstrated for optimal control of cardiac defibrillation with the presence of control constraints in a 3D rabbit ventricle geometries. We present the numerical results using different arrangement of control domain support at boundary of the computational domain. In the first test case, the feasible study of successful cardiac defibrillation is investigated where stimulus acts in the two large electrodes at the boundary of the bath domain. In the second test case we study the robustness of the optimization algorithm using variations in the conductivity tensors and model parameters and the convergence of the optimization 

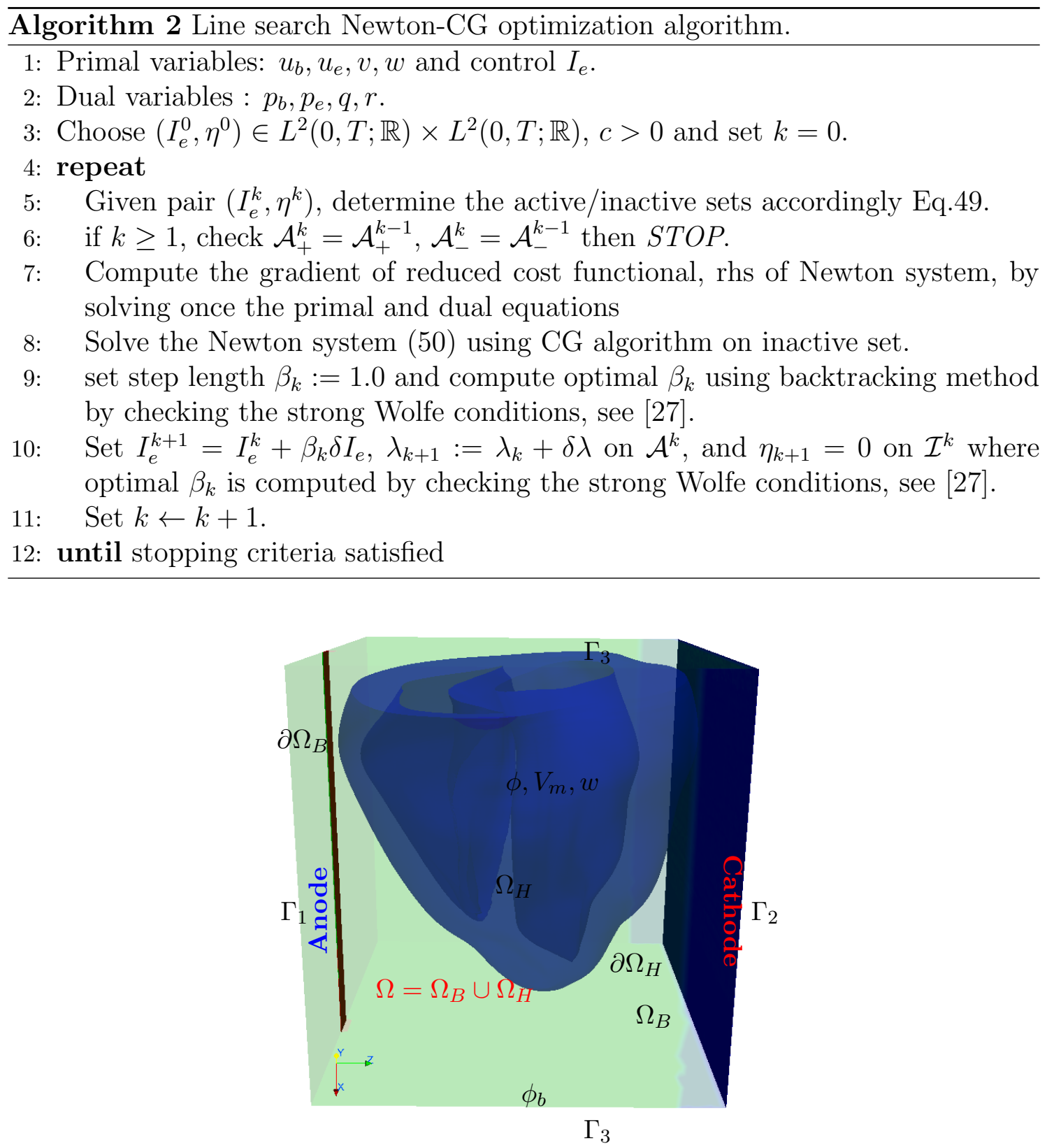

Figure 1: Schematic picture of the computational domain.

is presented on finer computational grids. In the third test case, the applicability of the multiple smaller boundary control domain support is investigated. At the end, the parallel efficiency is demonstrated.

In our computations, full three spatial dimensional rabbit ventricle geometry is considered which is generated based on the histological images [28]. The size of the integrated domain, $\Omega=\Omega_{H} \cup \Omega_{B}$, is $2.91 \times 3.12 \times 2.8 \mathrm{~cm}^{3}$. The computational domain of the integrated geometry comprises of 5,082,272 tetrahedral elements and 862,515 nodal points. The embedded cardiac tissue domain consists of 3,073,529 tetrahedrons and 547,680 nodal points. The computational domain and various relevant subdomains are depicted in Figure 1.

The conductivity values were chosen to arrive at physiologically relevant conduction velocities of $0.64 \mathrm{~m} / \mathrm{s}$ and $0.41 \mathrm{~m} / \mathrm{s}$ along and transverse to the principal fiber axes, respectively, and to keep anisotropy ratios within the range of values reported in experimental studies [32. A rule-based method was used to impose fiber 
orientations using fiber angles of $-60 \circ$ and $+60 \circ$ at the endocardial and epicardial surfaces, respectively, and a smooth linear variation of fiber angles as a function of depth in between. The glyph of the fiber directions $a_{l}(x)$ at the cardiac tissue is shown in Figure 2 which are used to compute the anisotropic conductivity tensor values accordingly Eq.(5).

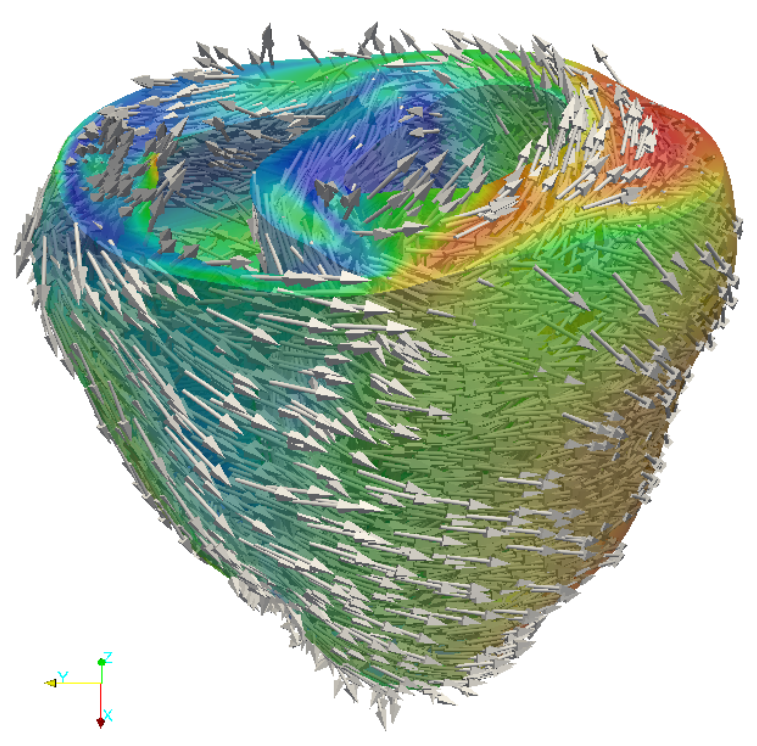

Figure 2: The glyph of the fiber directions $a_{l}(x)$.

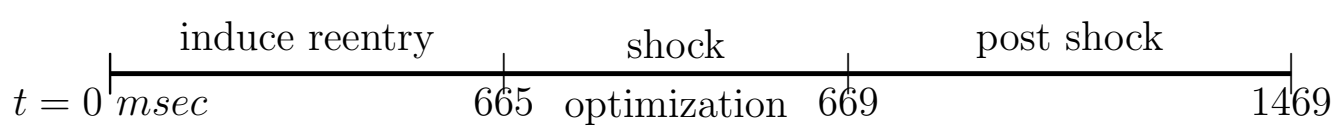

Figure 3: Different time horizons considered in the computations.

We followed a standard $S 1-S 2$ stimulation protocol to induce the reentry at the computational domain, for more details see [12]. Brief overview of the three temporal horizons is depicted in Figure 3 to induce the reentry, applied shock strength duration and post shock simulation duration. The initial state solution of the extracellular potential, the transmembrane voltage and the gating variable for the optimization algorithm at time $t=665 \mathrm{msec}$ are depicted in Figure 4 .

To obtain a successful defibrillation, the desired trajectory of the transmembrane potential $\left(v_{d}\right)$ in objective function Eq. (31) plays a significant role. Accordingly, a solution of the primal problem was generated using a prescribed time course of stimulation current $I_{e}(t)=6 \mathrm{~mA} / \mathrm{cm}^{3}$ lasting $4 \mathrm{msec}$. The resulting desired trajectory ensures that optimized states attain a steady state during the post shock period. The aim of the optimal control procedure is to determine an optimal stimulus which still leads to successful defibrillation but with less energy than that used to $v_{d}$. In all test cases, the weight of at the observation domain is fixed $\alpha_{1}=0.05$ and the weight of the control cost is investigated. Concerning the choice of the parameters $\alpha_{1}$ and $\alpha_{2}$ in Eq. (31), let us note that the optimal control only depends on the ratio $\frac{\alpha_{2}}{\alpha_{1}}$. In the optimization procedure $\alpha_{1}$ acts as a scaling of the dual variables 


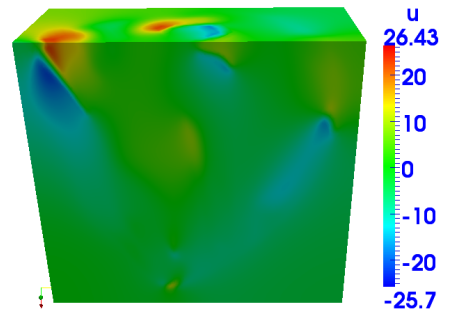

(a) extracellular potential

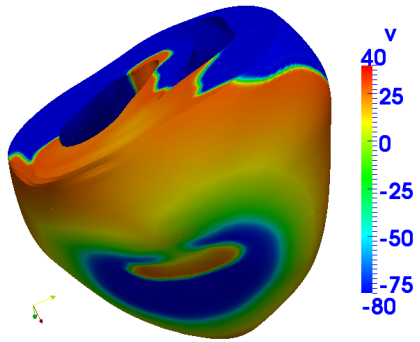

(b) transmembrane voltage

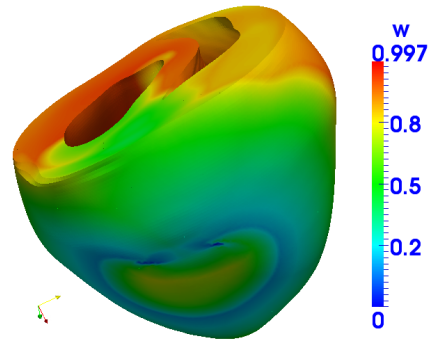

(c) gating variable

Figure 4: The solution $(u, v, w)$ at $t=585$ msec.

$\left(p_{b}, p, q, r\right)$. For fixed $\alpha_{1}$, the weight $\alpha_{2}$ describes the relative weight of the cost of the control $I_{e}$. In our computation we set $\alpha_{1}=0.05$ and analyze the effect of varying $\alpha_{2}$. The complete optimization code was developed based on the public domain FEM software package DUNE 3. The presented numerical results are done on a Linux cluster consisting of ten nodes where each node consists of 8 quad-core AMD Opteron processors 8356 clocked at $2.3 \mathrm{GHz}$ and equipped with 1TB RAM.

\subsection{Cardiac defibrillation}

Here we give the numerical results for the successful defibrillation by utilizing less applied current. For this simulations, the desired trajectory solution is constructed by applying the external stimulus strength $I_{e}(t)=6 \mathrm{~mA} / \mathrm{cm}^{3}$ over the $4 \mathrm{msec}$ of simulation time. In optimization, we impose the bilateral control constraints as $-6 \leq \bar{I}_{e}(t) \leq 6$.

The $\mathrm{L}^{2}$ norm of the gradient and minimization value of the cost functional over the optimization iterations for different regularization parameter values are depicted in Figure 5 ( $\log$ scale at Y-axis).

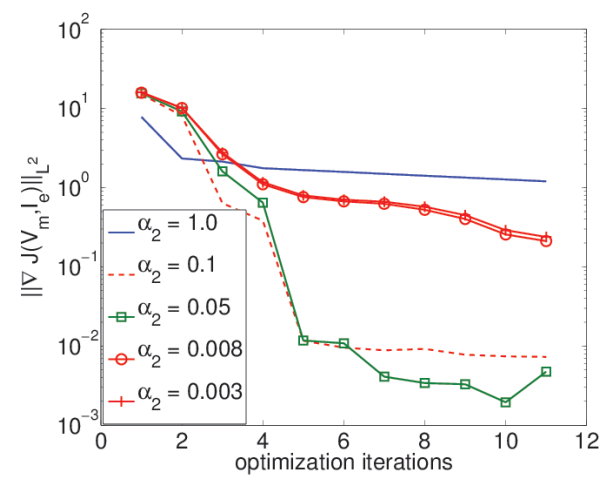

(a) $\mathrm{L}^{2}$ norm of the gradient of $J$

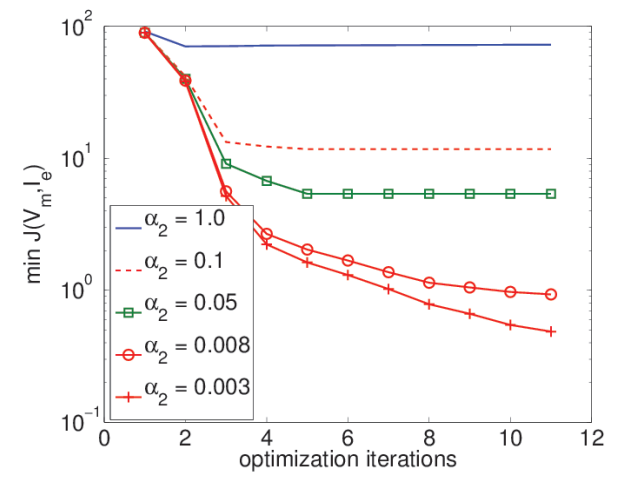

(b) minimization value of $J$

Figure 5: The solution $(u, v, w)$ at $t=585$ msec.

The optimal control value over the time for different weights of the costs is depicted in Figure 6. We observed that successful defibrillation is observed during the post shock simulations except for $\alpha_{2}=1.0$. We can observe that the control constraints are getting active over the time period as the regularization parameter is decreased. The total current is $21.7229,21.4031,18.6085,16.1175$ and 5.6407 $\mathrm{mA} / \mathrm{cm}^{3}$ respectively for $\alpha_{2}=0.003,0.008,0.05,0.1$ and 1 . 


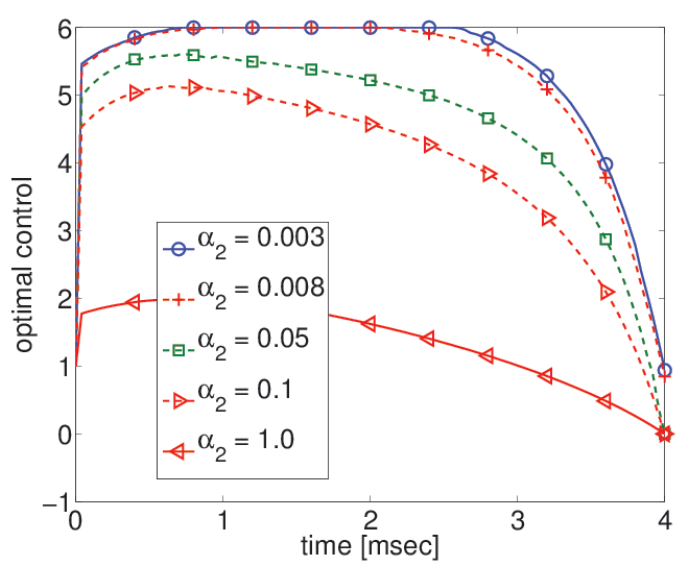

Figure 6: The optimal control.

The uncontrolled transmembrane voltage solution is depicted for various time instances in Figure 7. The tip of the reentrant source is moving around the computational domain and as a consequence the excitation wave fronts appear all the time on the computational domain. The corresponding optimal state solution and the postshock solution are shown in Figure 8 for different time steps. Here we can observe that during the initial shock period, there are many virtual electrodes at time $t=665.4$. From this point on, during the shock period, the virtual electrode polarization strength increases in the vicinity of the initial polarization. Due to sufficient mass of virtual electrode polarization and small excitability gap, the existed reentrant wave did not survive to propagate further excitation wavefronts. We can observe that the reentrant phenomena has completely disappeared at time 1340 msec.
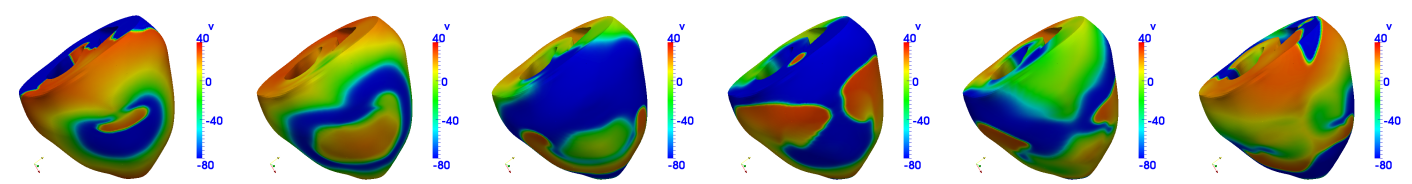

Figure 7: The uncontrolled solution of transmembrane voltage $v$ at times $t=665.40$, 7611.90, 866.40, 931.0, 1195.2 and 1331.90 msecs respectively.
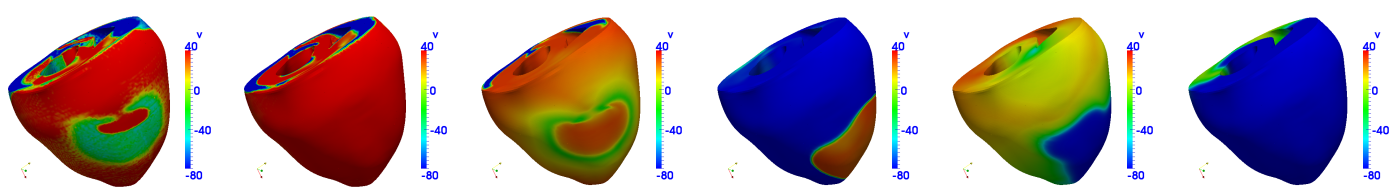

Figure 8: The optimal state solution of transmembrane voltage $v$ during the shock period at times $t=665.40$ and 669 msecs in first two panels. In last 4 panels, the solution of $v$ during the post-shock period at times $t=673.15,931.0,1195.3$ and $1331.0 \mathrm{msec}$ respectively. 


\subsection{Robustness w.r.t to variations in model parameters}

In this subsection, the robustness of the approach is demonstrated by introducing the variations in the model parameters as well as in the conductivity tensor values. Here we perform two test cases for the demonstration. In the first test case, the characteristic time of closing gate parameter is reduced by $10 \%$ in the simulations while the other parameters were kept unchanged. In both test cases, we choose the regularization parameter $\alpha_{2}=0.05$. Due to the reduction in $\tau_{\text {close }}$, the result of the APD duration is shortened from $330 \mathrm{msec}$ to $302 \mathrm{msec}$ in the simulations. The optimal control $\bar{I}_{e}(t)$ is depicted in Figure 9 (a) over the time with variations in the $\tau_{\text {close }}$ model parameter. The time course of the optimal control over the time looks very similar to the one presented in the above subsection. The computed total current is $18.6248 \mathrm{~mA} / \mathrm{cm}^{3}$ as opposed to the $18.6085 \mathrm{~mA} / \mathrm{cm}^{3}$. We point out that with the reduction of $15 \%$ in closing gate parameter, we did not see a successful defibrillation.

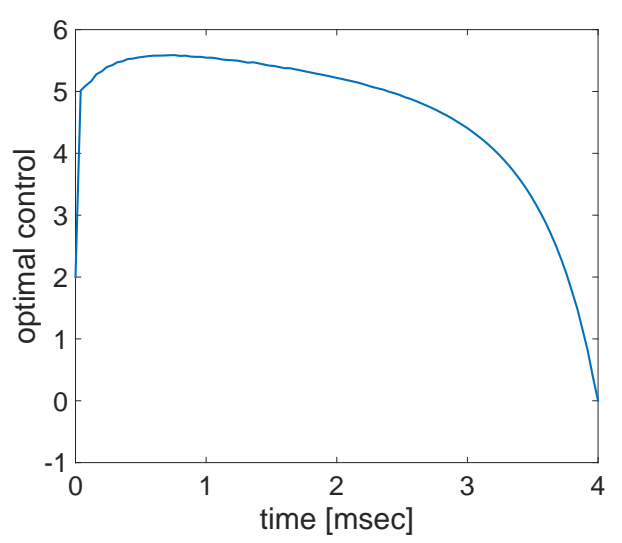

(a) for $\tau_{\text {close }}=135$

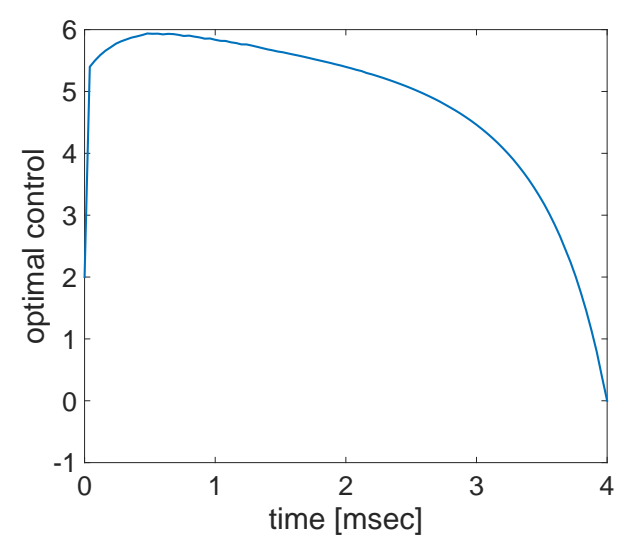

(b) for variation in conductivity tensors

Figure 9: The optimal control solution .

In the second test case, $10 \%$ of reduction is included in the conductivity tensor values which results in slowdown of the propagation of the wavefront in numerical simulations. The optimal control $\bar{I}_{e}(t)$ is depicted in Figure 9(b) over the time with variations in the conductivity tensor values. In this test case the computed total current is $19.3155 \mathrm{~mA} / \mathrm{cm}^{3}$. Both test cases are considered to test the successful defibrillation for different patient data by utilizing the pre-computed desired trajectory solution in our simulations.

\subsection{Convergence with finer grid}

The optimization algorithm convergence w.r.t. the fine resolution mesh is studied in this subsection. The global refinement of the coarse grid rabbit ventricle geometry, shown in Figure 1, mesh is called the finer mesh in our computations. In it the 
integrated domain consists of 40,658,176 tetrahedral elements and 6,828,862 nodes and the tissue geometry comprises of 24,588,232 tetrahedrons and 4,235,684 vertices. For this test case, the initial guess for the control is taken from the optimal control solution obtained for the test case with $\alpha_{2}=0.05$ in Figure 6 .

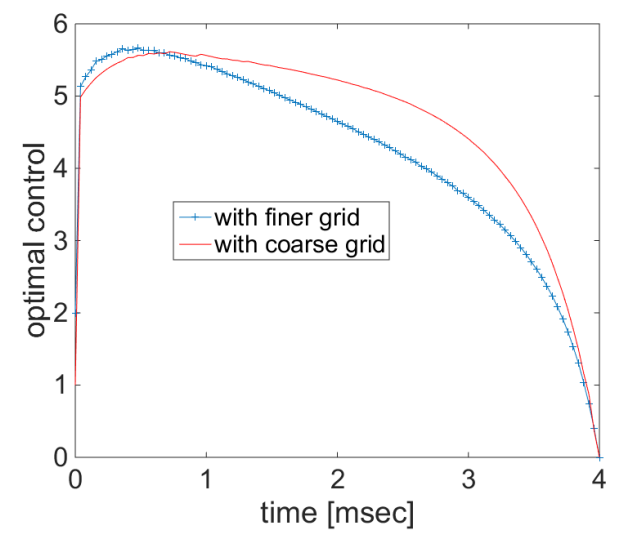

Figure 10: The optimal control solution on the finer and coarse grid geometries.

We observed that the Newton-CG optimization algorithm has taken 4 iterations to converge the solution. The optimal control solution is shown in Figure 10, In this finer grid computation case, the optimal control value is higher at the beginning of the time and then it is smaller as compared to coarse grid computations, see Figure 6 for the case of $\alpha=0.05$. The total current is $17.0771 \mathrm{~mA} / \mathrm{cm}^{3}$ and we can observe that the finer grid computation takes less total current compare to coarse grid which was $18.6085 \mathrm{~mA} / \mathrm{cm}^{3}$.

\subsection{Multiple boundary control domains}

In this subsection numerical results are presented based on the smaller support of the control domains at the boundary of the computational domain as shown in Figure 11. The local support of the anode boundary regions are placed on three lateral boundary surfaces and the local support of the cathode boundary regions is placed along one boundary surface of the computational domain. For practical relevance, it is desirable that multiple injected current boundary support be as small as possible. In this case, the desired trajectory is generated by applying the external stimulus strength $I_{e}(t)=6 \mathrm{~mA} / \mathrm{cm}^{3}$ in this test case as well. The weight of the cost of the control is reduced to $\alpha_{2}=0.001$ in order to obtain successful defibrillation. Moreover, a larger than this value did not lead to a successful defibrillation.

We point out that the same experiment was carried with anode boundary regions only placed on top surface rather than on three surfaces. Due to lack of appearance of sufficiently many virtual electrodes and larger excitable gaps, this did not lead to a successful defibrillation.

The $\mathrm{L}^{2}$-norm of the gradient of the cost functional and optimal control solution is depicted in Figure 12. We can observe that the control constraints are active from $0.2 \mathrm{msec}$ to about $1 \mathrm{msec}$ of simulation time. The total current is $22.2178 \mathrm{~mA} / \mathrm{cm}^{3}$. In this case, we observed that the reentrant wave is disappeared at simulation time 1365 msec. 


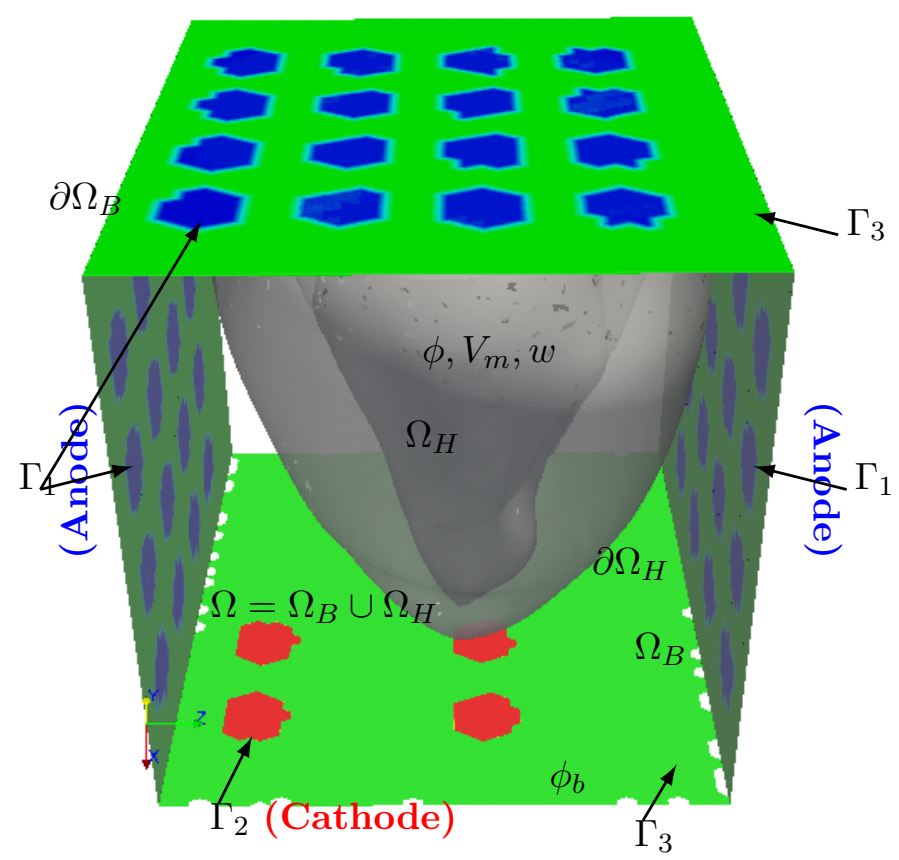

Figure 11: The computational domain with smaller control support at the boundary.
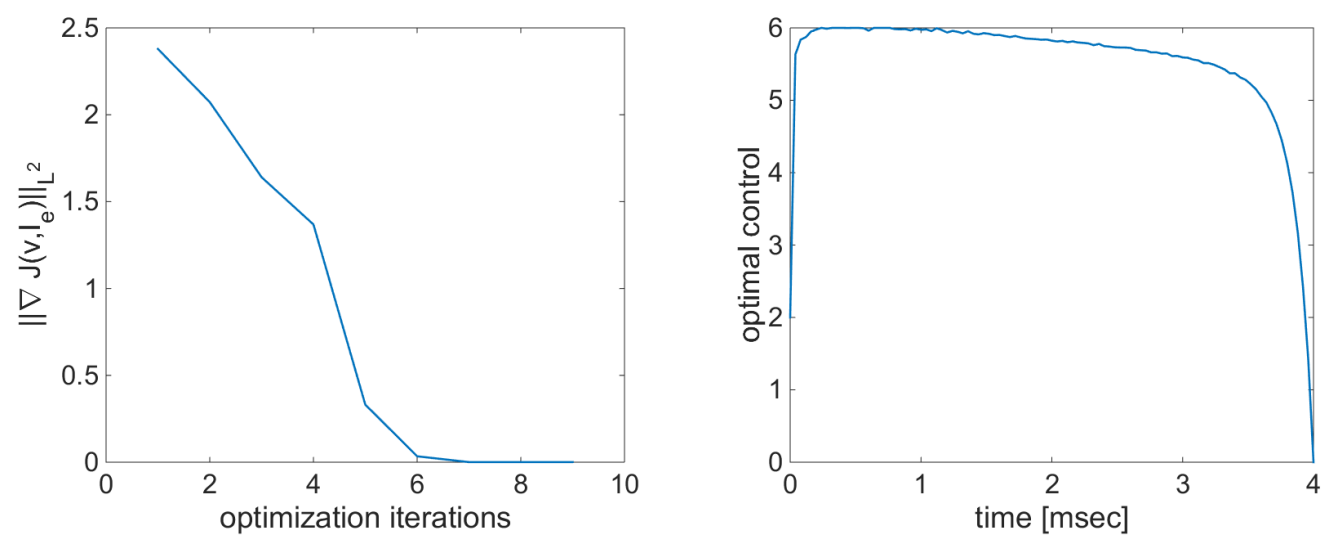

Figure 12: The $\mathrm{L}^{2}$-norm of the gradient of the cost functional at the left and the optimal control value over the time at the right.

\subsection{Parallel efficiency}

To achieve significant reduction in computational time it is key to optimize solvers in terms of strong scalability of the partial differential equations which dominate the overall computational cost in an optimal control solver. Benchmarking parallel scalability of the optimization algorithm was performed using a high resolution mesh (minimum edge size is $h=1.8533$ ), which is demonstrated in Section 4.3 , to assess the strong scaling properties of the solvers. The strong parallel efficiency was computed as

$$
e=\frac{N_{r}}{N} \frac{T_{r}}{T_{N}}
$$

where $N_{r}$ and $T_{r}$ are number of cores and total cpu time of a reference simulation, respectively, and $N$ and $T_{N}$ are number of cores and total cpu time in the scalability experiments. We have chosen $N_{r}=4$ in this test case due to too long computational 
time on single cpu.

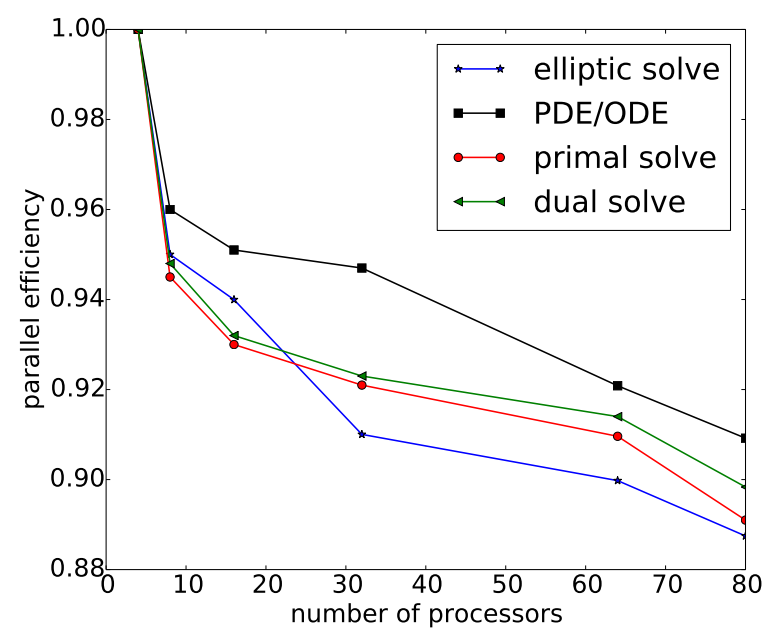

Figure 13: Parallel efficiency for different components of the optimization algorithm.

Benchmark results are illustrated in Fig. 13 for different components of bidomain solver. The parallel efficiency is calculated based on the first iteration of optimization algorithm. First we discuss the absolute times at the reference simulation. The cpu times for elliptic, PDE/ODE, primal and dual solves require 9156 minutes, 4470 minutes, $13670 \mathrm{~min}$, and 12156 minutes. In computations, due to the solution strategy of bidomain system, we observed that the communication time for sending the elliptic solution from the whole domain to the tissue domain taken 48.4 seconds and 56.3 seconds for communicating the transmembrane solution from the tissue domain to the whole domain. The complete optimization took approximately 3 days 14 hours on 80 cores to complete optimization algorithm. We can observe that the parallel efficiency of $e=0.89$ could be achieved for the primal and dual solves on 80 cores.

\section{Summary}

In the present work, we demonstrated the efficient primal dual active set methods for the optimization of bidomain equations on realistic 3D anatomical rabbit ventricle geometries. We extended our earlier study [12] by considering the realistic 3D anatomical geometries, in the presence of the bilateral control constraints to circumvent the excess injected currents. In our computations, we adapted a primal-dual active set [18] strategy to accommodate the control constraints in the optimization algorithm. In the numerical experiments we observed that the control constraints are active when the weight of the cost of control is getting smaller.

We studied the possibility of successful defibrillation on such realistic 3D anatomical geometries with considering the phenomenological model, Mitchell-Schaeffer model by delivering the strong shocks at the boundary of the bath domain. In our computations, we considered this served as a reference solution for optimization algorithm. Optimal control techniques demonstrated that the successful defibrillation is possible with less total current comparing with the ad-hoc strategy.

We demonstrated the robustness of the optimization algorithm by modifying the model parameters and conductivity tensors values. This can be attributed as 
using a pre-existing trajectory solution which was computed based on existing data and testing the successful defibrillation for different patient data. Moreover, we conducted the convergence study of the finer meshes for the demonstration of the robustness of the optimization algorithm. In this case the finer grid computation takes less injected current than the coarser grid computation. In our numerical experiment we investigated the applicability of the multiple smaller boundary control support and demonstrated that successful defibrillation can be achieved. In this case, the disappearance of reentrant wave took more time than the appearance of control at the full boundary surface. The good parallel efficiency is accomplished for the primal-dual active set strategy for such complex geometries on 80 cores.

\section{Acknowledgment}

The authors gratefully acknowledge the Austrian Academy of Sciences (OÄW).

\section{References}

[1] CellML Model Repository. http://models.cellml.org/cellml.

[2] T. Austin, M. L. Trew, and A. Pullan. Solving the cardiac bidomain equations for discontinuous conductivities. Biomedical Engineering, IEEE Transactions on, 53(7):1265-1272, July 2006.

[3] P. Bastian, M. Blatt, A. Dedner, C. Engwer, R. Klöfkorn, R. Kornhuber, M. Ohlberger, and O. Sander. A generic grid interface for parallel and adaptive scientific computing. Part II: implementation and tests in DUNE. Computing, 82(2):121-138, July 2008.

[4] P. Bastian, F. Heimann, and S. Marnach. Generic implementation of finite element methods in the distributed and unified numerics environment (DUNE). Kybernetika, 46(2):294-315, 2010.

[5] J. D. Bayer, R. C. Blake, G. Plank, and N. A. Trayanova. A novel rule-based algorithm for assigning myocardial fiber orientation to computational heart models. Ann Biomed Eng, 40(10):2243-2254, Oct 2012.

[6] M. Bergounioux, K. Ito, and K. Kunisch. Primal-dual strategy for constrained optimal control problems. SIAM J. Control Optim., 37(4):1176-1194, 1999.

[7] M. Blatt. A parallel algebraic multigrid method for elliptic problems with highly discontinuous coefficients. PhD thesis, Ruprechts-Karls-Universität Heidelberg, 2010 .

[8] P. Bochev and R. B. Lehoucq. On the finite element solution of the pure neumann problem. SIAM Rev., 47:50-66, January 2005.

[9] M. Boulakia, M. A. Fernández, J.-F. Gerbeau, and N. Zemzemi. A coupled system of PDEs and ODEs arising in electrocardiograms modeling. Applied Mathematics Research eXpress, 2008, 2008. 
[10] N. Chamakuri, , K. Kunisch, and G. Plank. Optimal control approach to termination of re-entry waves in cardiac electrophysiology. Journal of Mathematical Biology, 67(2):359-388, 2013.

[11] N. Chamakuri, K. Kunisch, and G. Plank. On boundary stimulation and optimal boundary control of the bidomain equations. Mathematical Biosciences, $245(2): 206-215,2013$.

[12] N. Chamakuri, K. Kunisch, and G. Plank. PDE constrained optimization of electrical defibrillation in a 3d ventricular slice geometry. International Journal of Numerical Methods in Biomedical Engineering (in revision), 2015.

[13] Y. Cheng, K. A. Mowrey, D. R. Van Wagoner, P. J. Tchou, and I. R. Efimov. Virtual electrodeinduced reexcitation: A mechanism of defibrillation. Circulation Research, 85(11):1056-1066, 1999.

[14] A. Dedner, C. Rohde, B. Schupp, and M. Wesenberg. A parallel, load-balanced mhd code on locally-adapted, unstructured grids in 3d. Computing and Visualization in Science, 7(2):79-96, 2004.

[15] M. Ethier and Y. Bourgault. Semi-implicit time-discretization schemes for the bidomain model. SIAM Journal on Numerical Analysis, 46(5):2443-2468, 2008.

[16] P. C. Franzone, P. Deuflhard, B. Erdmann, J. Lang, and L. F. Pavarino. Adaptivity in space and time for reaction-diffusion systems in electrocardiology. SIAM Journal on Numerical Analysis, 28(3):942-962, 2006.

[17] M. Hintermüller. A primal-dual active set algorithm for bilaterally control constrained optimal control problems. Quarterly of Applied Mathematics, 61:725746, 2003.

[18] M. Hintermüller, K. Ito, and K. Kunisch. The primal-dual active set strategy as a semismooth newton method. SIAM J. on Optimization, 13(3):865-888, 2002.

[19] D. A. Hooks, K. A. Tomlinson, S. G. Marsden, I. J. LeGrice, B. H. Smaill, A. J. Pullan, and P. J. Hunter. Cardiac microstructure: Implications for electrical propagation and defibrillation in the heart. Circulation Research, 91(4):331$338,2002$.

[20] K. Ito and K. Kunisch. The primal-dual active set method for nonlinear optimal control problems with bilateral constraints. SIAM J. Control and Optimization, 43(1):357-376, 2004.

[21] K. Ito and K. Kunisch. Lagrange Multiplier Approach to Variational Problems and Applications. Society for Industrial and Applied Mathematics, Philadelphia, PA, USA, 2008.

[22] G. Karypis and V. Kumar. A fast and high quality multilevel scheme for partitioning irregular graphs. SIAM Journal on Scientific Computing, 20(1):359392, 1998. 
[23] J. P. Keener. Modeling electrical activity of cardiac cells, two variable models. www.math.utah.edu/ keener/lectures/ionic_models/Two_variable_ models.

[24] J. P. Keener and K. Bogar. A numerical method for the solution of the bidomain equations in cardiac tissue. Chaos: An Interdisciplinary Journal of Nonlinear Science, 8(1):234-241, 1998.

[25] J. Lang. Adaptive Multilevel Solution of Nonlinear Parabolic PDE Systems, volume 16 of Lecture Notes in Computational Science and Engineering. SpringerVerlag, Berlin, 2001.

[26] C. Mitchell and D. Schaeffer. A two-current model for the dynamics of cardiac membrane. Bulletin of Mathematical Biology, 65(5):767-793, 2003.

[27] J. Nocedal and S. J. Wright. Numerical Optimization. Springer Verlag, New York, second edition edition, 2006.

[28] G. Plank, R. A. Burton, P. Hales, M. Bishop, T. Mansoori, M. O. Bernabeu, A. Garny, A. J. Prassl, C. Bollensdorff, F. Mason, F. Mahmood, B. Rodriguez, V. Grau, J. A. E. Schneider, D. Gavaghan, and P. Kohl. Generation of histoanatomically representative models of the individual heart: tools and application. Philosophical Transactions of the Royal Society A: Mathematical, Physical and Engineering Sciences, 367(1896):2257-2292, 2009.

[29] G. Plank, M. Liebmann, R. W. dos Santos, E. Vigmond, and G. Haase. Algebraic multigrid preconditioner for the cardiac bidomain model. IEEE Trans Biomed Eng., 54(4):585-596, 2007.

[30] R. Plonsey. Bioelectric sources arising in excitable fibers (ALZA lecture). Ann Biomed Eng, 16(6):519-46, 1988.

[31] M. Potse, B. Dube, J. Richer, A. Vinet, and R. Gulrajani. A comparison of monodomain and bidomain reaction-diffusion models for action potential propagation in the human heart. IEEE Transactions on Biomedical Engineering, 53(12):2425-2435, Dec. 2006.

[32] B. J. Roth. Electrical conductivity values used with the bidomain model of cardiac tissue. IEEE Trans Biomed Eng, 44(4):326-328, Apr 1997.

[33] N. Trayanova, J. Constantino, T. Ashihara, and G. Plank. Modeling defibrillation of the heart: approaches and insights. IEEE reviews in biomedical engineering, 4:89-102, 2011.

[34] N. Trayanova, J. Eason, and F. Aguel. Computer simulations of cardiac defibrillation: a look inside the heart. Computing and Visualization in Science, 4(4):259-270, 2002.

[35] L. Tung. A bi-domain model for describing ischemic myocardial DC potentials. PhD thesis, MIT, Cambridge, MA, 1978.

[36] M. Ulbrich. Semismooth newton methods for operator equations in function spaces. SIAM Journal on Optimization, 13(3):805-841, 2002. 